\title{
Tutte's 5-flow conjecture for highly cyclically connected cubic graphs
}

\author{
Eckhard Steffen \\ Paderborn Institute for Advanced Studies in Computer Science and Engineering, \\ Universität Paderborn, Warburger Straße 100, D-33098 Paderborn, Germany
}

In 1954, Tutte conjectured that every bridgeless graph has a nowhere-zero 5-flow. Let $\omega(G)$ be the minimum number of odd cycles in a 2-factor of a bridgeless cubic graph $G$. Tutte's conjecture is equivalent to its restriction to cubic graphs with $\omega \geq 2$. We show that if a cubic graph $G$ has no edge cut with fewer than $\frac{5}{2} \omega(G)-3$ edges that separates two odd cycles of a minimum 2 -factor of $G$, then $G$ has a nowhere-zero 5 -flow. This implies that if a cubic graph $G$ is cyclically $n$-edge connected and $n \geq \frac{5}{2} \omega(G)-3$, then $G$ has a nowhere-zero 5-flow.

\section{Introduction}

This paper is about flows on finite graphs. Let $M=(V, E)$ be a graph with vertex set $V$ and edge set $E$. Each edge is incident to precisely two different vertices, i.e. multiple edges may occur but there are no loops.

An orientation $D$ of $M$ is an assignment of a direction to each edge, and for $v \in V$, $D^{-}(v)\left(D^{+}(v)\right)$ is the set of edges whose head (tail) is incident to $v$. The oriented graph is denoted by $D(M), d_{D(M)}^{-}(v)=\left|D^{-}(v)\right|$ and $d_{D(M)}^{+}(v)=\left|D^{+}(v)\right|$ denote the indegree and outdegree of vertex $v$ in $D(M)$, respectively.

Let $k \geq 2$ be a positive integer and $\varphi: E \longrightarrow\{0,1, \ldots, k-1\}$ be a function. If for all $v \in V$,

$\sum_{e \in D^{+}(v)} \varphi(e)=\sum_{e \in D^{-}(v)} \varphi(e)$,

then $(D, \varphi)$ is a $k$-flow on $M$. If, in addition, $\varphi(e) \neq 0$, for all $e \in E$, then $(D, \varphi)$ is a nowhere-zero $k$-flow on $M$. In such a case, we say that $M$ has a nowhere-zero $k$-flow.

If a graph has a nowhere-zero $k$-flow, then it has one for every $k^{\prime} \geq k$. Tutte [8] proved that a graph $G$ has a nowhere-zero $k$-flow $(D, \varphi)$ if and only if it has a flow $\left(D^{\prime}, \varphi^{\prime}\right)$ such that for every edge $e,\left|\varphi^{\prime}(e)\right|$ is one of $1, \ldots, k-1$. Thus determining for which number $k$ a graph has a nowhere-zero $k$-flow is a problem about graphs, not directed graphs.

Tutte [9] raised the problem to determine the smallest number $k$ for which a graph has a nowhere-zero $k$-flow, and he formulated the 5-Flow Conjecture.

Conjecture 1 [9] Every bridgeless graph has a nowhere-zero 5-flow.

The 5-Flow Conjecture is equivalent to its restriction to cubic graphs, cf. [3]. By Petersen's theorem, every bridgeless cubic graph $G$ has a 2-factor and the oddness $\omega(G)$ 
is the minimum number of odd cycles in a 2-factor of $G$. Clearly, the oddness must be an even number, and it is well known (cf. [3]) that a cubic graph $G$ has a nowhere-zero 4 -flow if and only if it is edge 3-colorable (i.e. $\omega(G)=0$ ). Hence the 5-Flow Conjecture is equivalent to its restriction to bridgeless cubic graphs with $\omega \geq 2$.

Many papers deal with the structure of a possible counterexample to the 5-Flow Conjecture. A connected graph $G=(V, E)$ that contains two disjoint cycles is cyclically $n$-edge connected if there is no edge cut $E^{\prime} \subset E$ with fewer than $n$ edges such that two components of $G-E^{\prime}$ contain cycles. The maximum number $k$ so that $G$ is cyclically $k$-edge connected is the cyclic connectivity of $G$ and it is denoted by $n_{G}^{*}$. Kochol [4,5] showed that the length of a shortest cycle in a possible minimum counterexample is at least 9, and that it is cyclically 6 -edge connected. This paper proves the following theorems.

Theorem 1 Every cubic graph $G$ with cyclic connectivity $n_{G}^{*} \geq \frac{5}{2} \omega(G)-3$ has a nowherezero 5-flow.

A minimum 2-factor of a cubic graph $G=(V, E)$ has precisely $\omega(G)$ odd cycles. Let $\omega(G) \geq 2, \mathcal{F}_{2}$ be a minimum 2-factor, and let $m_{G}\left(\mathcal{F}_{2}\right)$ be the maximum number $k$ such that there is no edge cut $E^{\prime} \subset E$ with fewer than $k$ edges such that two components of $G-E^{\prime}$ contain odd cycles of $\mathcal{F}_{2}$. We define $m_{G}^{*}=\max \left\{m_{G}\left(\mathcal{F}_{2}\right) \mid \mathcal{F}_{2}\right.$ is a minimum 2-factor of $\left.G\right\}$ to be the cyclic factor connectivity of $G$. For graphs $G$ with $\omega(G)=0$ define $m_{G}^{*}=\infty$.

Since $n_{G}^{*} \leq m_{G}^{*}$ Theorem 11 is a direct consequence of the following theorem.

Theorem 2 Let $G$ be a bridgeless cubic graph. If $m_{G}^{*} \geq \frac{5}{2} \omega(G)-3$, then $G$ has a nowhere-zero 5-flow.

\section{Balanced valuations and flow partitions}

Bondy [1] and Jaeger 2] introduced the concept of balanced valuations. A balanced valuation of a graph $M=(V, E)$ is a function $w$ from the vertex set $V$ into the real numbers such that for all $X \subseteq V:\left|\sum_{v \in X} w(v)\right| \leq\left|\partial_{M}(X)\right|$, where $\partial_{M}(X)$ is the set of edges with precisely one end in $X$. For $v \in V$ let $d_{M}(v)$ be the degree of $v$ in the undirected graph $M$. The following theorem relates integer flows to balanced valuations.

Theorem 3 [2] Let $M=(V, E)$ be a graph with orientation $D$ and $k \geq 3$. Then $M$ has a nowhere-zero $k$-flow $(D, \varphi)$ if and only if there is a balanced valuation $w$ of $M$ with $w(v)=\frac{k}{k-2}\left(2 d_{D(M)}^{+}(v)-d_{M}(v)\right)$, for all $v \in V$.

In particular, Theorem 3 says that a cubic graph $G$ has a nowhere-zero 4-flow (nowherezero 5-flow) if and only if there is a balanced valuation of $G$ with values in $\{ \pm 2\}\left(\left\{ \pm \frac{5}{3}\right\}\right)$.

Let $M=(V, E)$ be a multigraph. If $X \subseteq E$, then $M[X]$ denotes the graph whose vertex set consists of all vertices of edges of $X$ and whose edge set is $X$. Likewise if $X \subseteq V$, then $M[X]$ is the graph whose vertex set is $X$ and whose edge set consists of those edges incident to two vertices of $X$. In both instances the subgraph $M[X]$ is called the subgraph of $M$ induced by $X$.

Let $E_{i} \subseteq E$, and $\left(D_{i}, \varphi_{i}\right)$ be flows on $M\left[E_{i}\right], i=1,2$. The $\operatorname{sum}\left(D_{1}, \varphi_{1}\right)+\left(D_{2}, \varphi_{2}\right)$ is the flow $(D, \varphi)$ on $M\left[E_{1} \cup E_{2}\right]$ with orientation

$D:=\left.\left.D_{1}\right|_{\left\{e \mid \varphi_{1}(e) \geq \varphi_{2}(e)\right\}} \cup D_{2}\right|_{\left\{e \mid \varphi_{2}(e)>\varphi_{1}(e)\right\}}$, and 
$\varphi(e):=\left\{\begin{array}{l}\varphi_{1}(e)+\varphi_{2}(e) \text { if } e \text { received the same direction in } D_{1} \text { and } D_{2} \\ \left|\varphi_{1}(e)-\varphi_{2}(e)\right| \text { otherwise, }\end{array}\right.$

for $e \in E_{1} \cup E_{2}$.

Let $G=(V, E)$ be a bridgeless cubic graph, and $\mathcal{F}_{2}$ be a 2-factor of $G$ with odd cycles $C_{1}, C_{2}, \ldots, C_{2 t}$, and even cycles $C_{2 t+1}, \ldots, C_{2 t+\ell}(t \geq 0, \ell \geq 0)$, and let $\mathcal{F}_{1}$ be the complementary 1-factor.

A canonical 4-coloring of $G$ (with respect to $\mathcal{F}_{2}$ ) colors the edges of $\mathcal{F}_{1}$ with color 1 , the edges of the even cycles with 2 and 3, alternately, and the edges of the odd cycles with colors 2 and 3 alternately, except one edge which is colored 0 . Then, there are precisely $2 t$ vertices $z_{1}, z_{2}, \ldots, z_{2 t}$ where color 2 is missing.

Let $M_{G}=\left(V, E\left(M_{G}\right)\right)$ be the graph obtained from $G$ by adding two edges $f_{i}$ and $f_{i}^{\prime}$ between $z_{2 i-1}$ and $z_{2 i}$ for $i=1, \ldots, t$. Extend $c$ to a proper edge coloring of $M_{G}$ by coloring $f_{i}^{\prime}$ with color 2 and $f_{i}$ with color 4 . Let $C_{1}^{\prime}, \ldots C_{s}^{\prime}$ be the 2 -factor of $M_{G}$ induced by the edges of colors 1 and $2(s \geq 1)$, and for $i=1, \ldots, t$ let $C_{i}^{\prime \prime}$ be the 2-cycle induced by the edges $f_{i}$ and $f_{i}^{\prime}$. We construct a nowhere-zero 4-flow on $M_{G}$ as follows:

For $1 \leq i \leq 2 t+\ell$ let $\left(D_{i}, \varphi_{i}\right)$ be a nowhere-zero flow on the directed cycle $C_{i}$ with $\varphi_{i}(e)=2$ for all $e \in E\left(C_{i}\right)$.

For $1 \leq i \leq s$ let $\left(D_{i}^{\prime}, \varphi_{i}^{\prime}\right)$ be a nowhere-zero flow on the directed cycle $C_{i}^{\prime}$ with $\varphi_{i}^{\prime}(e)=1$ for all $e \in E\left(C_{i}^{\prime}\right)$.

For $1 \leq i \leq t$ let $\left(D_{i}^{\prime \prime}, \varphi_{i}^{\prime \prime}\right)$ be a nowhere-zero flow on the directed cycle $C_{i}^{\prime \prime}$ (choose $D_{i}^{\prime \prime}$ such that $f_{i}^{\prime}$ receives the same direction as in $\left.D_{i}^{\prime}\right)$ with $\varphi_{i}^{\prime \prime}(e)=1$ for all $e \in\left\{f_{i}, f_{i}^{\prime}\right\}$. Then

$(D, \varphi)=\sum_{i=1}^{2 t+\ell}\left(D_{i}, \varphi_{i}\right)+\sum_{i=1}^{s}\left(D_{i}^{\prime}, \varphi_{i}^{\prime}\right)+\sum_{i=1}^{t}\left(D_{i}^{\prime \prime}, \varphi_{i}^{\prime \prime}\right)$

is a nowhere-zero 4 -flow on $M_{G}$.

By Theorem [3, there is a balanced valuation $w^{\prime}(v)=2\left(2 d_{D\left(M_{G}\right)}^{+}(v)-d_{M_{G}}(v)\right)$ of $M_{G}$. It holds that $\left|2 d_{D\left(M_{G}\right)}^{+}(v)-d_{M_{G}}(v)\right|=1$, and hence $w^{\prime}(v) \in\{ \pm 2\}$ for all $v \in V$. The vertices of $M_{G}$ (and therefore of $G$ as well) are partitioned into two classes $A=\left\{v \mid w^{\prime}(v)=-2\right\}$ and $B=\left\{v \mid w^{\prime}(v)=2\right\}$. Call the elements of $A(B)$ the white (black) vertices of $M_{G}$ and of $G$, respectively.

Let $G=(V, E)$ be a bridgeless cubic graph. A partition of $V$ into two classes $A$ and $B$ constructed as above, and using a 2-factor $\mathcal{F}_{2}$, a canonical 4-coloring $c$ of $G$, the 4-flow $(D, \varphi)$ on $M_{G}$ and the induced balanced valuation $w^{\prime}$ of $M_{G}$ is called a flow partition of $G$, and it is denoted by $P_{G}(A, B)=P_{G}\left(A, B, \mathcal{F}_{2}, c,(D, \varphi), w^{\prime}\right)$. If we refer to a special 2-factor $\mathcal{F}_{2}$, we say $P_{G}(A, B)$ is a flow partition of $G$ with respect to $\mathcal{F}_{2}$. For $X \subseteq V$ let $A_{X}=A \cap X\left(B_{X}=B \cap X\right)$ be the set of the white (black) vertices of $X$, and $a_{X}=\left|A_{X}\right|$, $b_{X}=\left|B_{X}\right|$. If we consider the vertex set $V(F)$ of a subgraph $F$ of a graph $G$ we also write $a_{F}$ instead of $a_{V(F)}\left(b_{F}\right.$ instead of $\left.b_{V(F)}\right)$.

We will prove some properties of flow partitions of cubic graphs. The following Lemma is a direct consequence of the construction of $(D, \varphi)$ on $M_{G}$.

Lemma 1 Let $P_{G}\left(A, B, \mathcal{F}_{2}, c,(D, \varphi), w^{\prime}\right)$ be a flow partition of a bridgeless cubic graph $G=(V, E)$, and $x y=e \in E$. If the canonical 4-coloring c colors $e$ with 1 or 2 , then $x$ and $y$ belong to different classes, i.e. $x \in A$ if and only if $y \in B$. 
Lemma 2 Let $G=(V, E)$ be a cubic bridgeless graph and $P_{G}(A, B)$ be a flow partition with respect to a 2-factor $\mathcal{F}_{2}$. Let $S \subseteq V$ be a set of vertices such that the induced subgraph $G[S]$ is connected, $n$ be the number of edges which have to be removed from $G[S]$ to obtain a spanning tree of $G[S]$, and let $n_{o}$ be the number of odd cycles of $\mathcal{F}_{2}$ which are subgraphs of $G[S]$. Then $b_{S} \leq 4 a_{S}+3-3 n+n_{o}$.

Proof. Let $P_{G}(A, B)=P_{G}\left(A, B, \mathcal{F}_{2}, c,(D, \varphi), w^{\prime}\right)$ and $F$ be a connected subgraph of $\mathcal{F}_{2}$. We show:

1) If $F$ is an even cycle, then $b_{F}=a_{F}$.

2) If $F$ is an odd cycle, then $b_{F} \leq a_{F}+1$.

3) If $F$ is a path, then $b_{F} \leq a_{F}+3$.

Items 1) and 2) follow from Lemma 1 directly. We distinguish two cases to prove 3).

Case 1: The edges of $F$ are colored with colors 2 and 3.

If $|E(F)|=2 l+1$, then at least $l$ edges are colored with color 2. Thus Lemma 1 implies that $a_{F} \geq l$. Since $|V(F)|=2 l+2$ and $b_{F}=2 l+2-a_{F}$ it follows that $b_{F} \leq a_{F}+2$.

If $|E(F)|=2 l$, then $l$ edges are colored with color 2 . Thus Lemma 1 implies that $a_{F}=l$. Since $|V(F)|=2 l+1$ it follows that $b_{F}=a_{F}+1$.

Case 2: $F$ contains an edge of color 0 .

By the definition of the coloring there is precisely one edge of color 0 .

If the length of $F$ is odd, say $2 l+1$, the first and the last edge of $F$ are colored differently, and there are $l$ edges of color 2. Thus Lemma 1 implies that $a_{F} \geq l$. Since $|V(F)|=2 l+2$ it follows that $b_{F} \leq a_{F}+2$.

If $|E(F)|=2 l$, then at least $l-1$ edges are colored 2. Thus Lemma 1 implies that $a_{F} \geq l-1$. Since $|V(F)|=2 l+1$ it follows that $b_{F} \leq a_{F}+3$. 。

Let $E_{1}$ be the set of edges of color 1 of $G[S]$. By Lemma 1, $\left|E_{1}\right| \leq a_{S}$. Let $E_{1}^{-} \subset E_{1}$ be a set of edges so that $G[S]-E_{1}^{-}$is connected and no edge of color 1 (in $G[S]-E_{1}^{-}$) is contained in a cycle. Each cycle of $G[S]-E_{1}^{-}$is a cycle of $\mathcal{F}_{2}$. Remove from each cycle precisely one edge of color 2 to obtain a spanning tree of $G[S]$. Let $E_{2}^{-}$be the set of these removed edges of color 2. With $n_{i}=\left|E_{i}^{-}\right|(i=1,2)$ it follows that $n=n_{1}+n_{2}$.

Let $Z_{0}, \ldots, Z_{a^{\prime}}$ be the components of $G[S]-E_{1}$, and $a_{i}\left(b_{i}\right)$ be the number of white (black) vertices in $Z_{i}, i=0, \ldots, a^{\prime}$. Each component is either a cycle of $\mathcal{F}_{2}$ or a subpath of a cycle of $\mathcal{F}_{2}$. The number of components is smaller than or equal to 1 plus the number of edges of color 1 in $G[S]-E_{1}^{-}$, therefore $a^{\prime} \leq a_{S}-n_{1}$. Furthermore $\sum_{i=0}^{a^{\prime}} a_{i}=a_{S}$.

For $i \in I_{P}=\left\{0,1, \ldots, a^{\prime}-n_{2}\right\}$ let $Z_{i}$ be a path, for $i \in I_{C}^{o}=\left\{a^{\prime}-n_{2}+1, \ldots, a^{\prime}-n_{2}+n_{o}\right\}$ let $Z_{i}$ be an odd cycle, and for $i \in I_{C}^{e}=\left\{a^{\prime}-n_{2}+n_{o}+1, \ldots, a^{\prime}\right\}$ let $Z_{i}$ be an even cycle. Then it follows with $a^{\prime} \leq a_{S}-n_{1}$ that

$$
\begin{aligned}
& b_{S}=\sum_{i \in I_{P}} b_{i}+\sum_{i \in I_{C}^{o}} b_{i}+\sum_{i \in I_{C}^{e}} b_{i} \\
\leq & \sum_{i \in I_{P}}\left(a_{i}+3\right)+\sum_{i \in I_{C}^{o}}\left(a_{i}+1\right)+\sum_{i \in I_{C}^{e}} a_{i} \\
= & 3\left(a^{\prime}-n_{2}+1\right)+n_{o}+\sum_{i=0}^{a^{\prime}} a_{i} \\
\leq & 3\left(a_{S}-\left(n_{1}+n_{2}\right)+1\right)+n_{o}+a_{S} \\
= & 4 a_{S}+3-3 n+n_{o} .
\end{aligned}
$$

We finish this section with the following lemma. 
Lemma 3 Let $P_{G}(A, B)$ be a flow partition of a cubic bridgeless graph $G=(V, E)$. Let $S \subseteq V$ be a set of vertices such that the induced subgraph $G[S]$ is connected, and $n$ be the number of edges which have to be removed from $G[S]$ to obtain a spanning tree $T$ of $G[S]$. Assume $a_{S} \leq b_{S}$, then $b_{S} \leq 4 a_{S}+3-3 n$ if and only if $\frac{5}{3}\left(b_{S}-a_{S}\right) \leq\left|\partial_{G}(S)\right|$.

Proof. Consider a spanning tree $T=(S, E(T))$ of $G[S]$ and let $T_{i}=\{v \mid v \in S$ and $\left.d_{T}(v)=i\right\}$, for $i=1,2$. Then $\left|\partial_{G}(S)\right|+2 n=2\left|T_{1}\right|+\left|T_{2}\right|$ and

$|S|-1=|E(T)|=\frac{1}{2}\left(3\left(|S|-\left(\left|T_{1}\right|+\left|T_{2}\right|\right)\right)+2\left|T_{2}\right|+\left|T_{1}\right|\right)=\frac{1}{2}\left(3|S|-\left|\partial_{G}(S)\right|-2 n\right)$.

Since $|S|=a_{S}+b_{S}$ it follows that $\left|\partial_{G}(S)\right|=a_{S}+b_{S}+2-2 n$, and hence $\frac{5}{3}\left(b_{S}-a_{S}\right) \leq$ $\left|\partial_{G}(S)\right|$ is equivalent to $b_{S} \leq 4 a_{S}+3-3 n$.

\section{Proof of Theorem 2}

Let $G=(V, E)$ be a bridgeless cubic graph with oddness $\omega$. If $\omega \in\{0,2\}$, then $G$ has a nowhere-zero 5 -flow, cf. [3]. Thus we may assume that $\omega \geq 4$.

Let $\mathcal{F}_{2}$ be a minimum 2-factor of $G$ with $m_{G}\left(\mathcal{F}_{2}\right)=m_{G}^{*} \geq \frac{5}{2} \omega-3$. Let $P_{G}(A, B)=$ $P_{G}\left(A, B, \mathcal{F}_{2}, c,(D, \varphi), w^{\prime}\right)$ be a flow partition of $G$ with respect to $\mathcal{F}_{2}$. Let $w: V \rightarrow\left\{ \pm \frac{5}{3}\right\}$ be a function with $w(v)=-\frac{5}{3}$ if $v \in A$ and $w(v)=\frac{5}{3}$ if $v \in B$. We will show that $w$ is a balanced valuation of $G$. Then it follows from Theorem 3 that $G$ has a nowhere-zero 5 -flow.

Assume to the contrary that $w$ is not balanced. Then there is $S \subseteq V$ with

$$
\left|\sum_{v \in S} w(v)\right|>\left|\partial_{G}(S)\right|
$$

If $S=V$, then $\left|\sum_{v \in S} w(v)\right|=0=\left|\partial_{G}(S)\right|$, and therefore $S$ is a proper subset of $V$. Let $S$ be of minimum order, so we may assume that $G[S]$ is connected, and without loss of generality $b_{S} \geq a_{S}$. With $k=b_{S}-a_{S}$ equation (3) becomes

$\frac{5}{3} k>\left|\partial_{G}(S)\right|$.

We show

Proposition $1\left|\partial_{G}(S)\right| \leq \frac{5}{2} \omega-4$; in particular

1) $\left|\partial_{G}(S)\right| \leq \frac{5}{2} \omega-4$, if $\left|\partial_{G}(S)\right| \equiv 1 \bmod 5$,

2) $\left|\partial_{G}(S)\right| \leq \frac{5}{2} \omega-8$, if $\left|\partial_{G}(S)\right| \equiv 2 \bmod 5$,

3) $\left|\partial_{G}(S)\right| \leq \frac{5}{2} \omega-7$, if $\left|\partial_{G}(S)\right| \equiv 3 \bmod 5$,

4) $\left|\partial_{G}(S)\right| \leq \frac{5}{2} \omega-11$, if $\left|\partial_{G}(S)\right| \equiv 4 \bmod 5$,

5) $\left|\partial_{G}(S)\right| \leq \frac{5}{2} \omega-15$, if $\left|\partial_{G}(S)\right| \equiv 0 \bmod 5$.

Proof. For $i=0,1,2,3$ let $E_{i} \subset E$ be the set of the edges of color $i$ in $G$ and let $c_{i}=\left|\partial_{G}(S) \cap E_{i}\right|$. The edges of color 1 form a 1-factor of $G$. Thus Lemma 1 implies that $k=c_{1}$ and hence $c_{1}>\frac{3}{5}\left|\partial_{G}(S)\right|$ by equation (4). 
Let $l_{S}^{a}\left(l_{S}^{b}\right)$ be the number of white (black) vertices of $S$ where color 2 is missing, with respect to $c$. Let $l=\left|l_{S}^{b}-l_{S}^{a}\right|$. From $0 \leq l_{S}^{a}, l_{S}^{b} \leq \frac{1}{2} \omega$ it follows that $l \leq \frac{1}{2} \omega$, and Lemma 1 implies that $k \leq c_{2}+l$. Hence $c_{2}+\frac{1}{2} \omega \geq k>\frac{3}{5}\left|\partial_{G}(S)\right|$.

1) If $\left|\partial_{G}(S)\right| \equiv 1 \bmod 5$, say $\left|\partial_{G}(S)\right|=5 m+1$, then it follows that $c_{1} \geq 3 m+1$ and therefore $c_{2} \leq 2 m$. Thus $\frac{1}{2} \omega \geq 3 m+1-c_{2} \geq 3 m+1-2 m=m+1$ and hence $\frac{5}{2} \omega-4 \geq\left|\partial_{G}(S)\right|$.

2) can be proved analogously.

3) If $\left|\partial_{G}(S)\right| \equiv 3 \bmod 5$, say $\left|\partial_{G}(S)\right|=5 m+3$, then it follows that $c_{1} \geq 3 m+2$ and therefore $c_{2} \leq 2 m+1$.

If $c_{2}=2 m+1$, then $c_{1} \leq\left|\partial_{G}(S)\right|-c_{2}=3 m+2$ and hence $c_{1}=3 m+2$ and $c_{0}=c_{3}=0$. Let $X$ be the set of vertices of $G[S]$ which are incident (in $G$ ) to an edge of $\left|\partial_{G}(S) \cap E_{2}\right|$, and $Y$ be the set of vertices which are incident to an edge of color 0 in $G[S]$. Color 2 or 3 is missing on each vertex of $X \cup Y$ and $Z=X \cap Y$ consists of those vertices of $G[S]$ where both colors, 2 and 3, are missing. Each vertex $z$ of $Z$ is incident to an edge $e=z z^{\prime}$ of color 0 in $G[S]$. Furthermore, color 2 is missing and color 3 appears at $z^{\prime}$. Therefore, for each vertex of $z \in Z$ there is precisely one vertex $z^{\prime}$ in $G[S]$ where only color 2 is missing. Since $|X|=c_{2}$ is odd and $c_{0}=c_{3}=0$ it follows that the total number of vertices of $G[S]$ where either color 2 or color 3 is missing is odd. This is a contradiction, since every path induced by edges of colors 2 and 3 in $G[S]$ has precisely two end vertices in $G[S]$.

Therefore $c_{2} \leq 2 m$ and hence $c_{2}+\frac{1}{2} \omega \geq 3 m+2$ implies that $\frac{1}{2} \omega \geq 3 m+2-2 m=m+2$. Thus $\frac{5}{2} \omega-7 \geq 5 m+3=\left|\partial_{G}(S)\right|$.

Items 4) and 5) can be proved analogously to 3). $\circ$

Since $G$ has no edge cut with fewer than $\frac{5}{2} \omega-3$ edges that separates two odd cycles of $\mathcal{F}_{2}$ it follows with Proposition 1 that $n_{o}=0$. Hence $b_{S} \leq 4 a_{S}+3-3 n$ by Lemma 2 and therefore $\frac{5}{3} k \leq\left|\partial_{G}(S)\right|$ by Lemma 3. This contradicts equation (4) and completes the proof.

\section{Remarks on $r$-flows}

The notion of nowhere-zero flows can be extended to rational numbers. Let $1 \leq p \leq q$ be integers, and let $\varphi$ be a function from the edge set $E$ of the directed graph $G=(V, E)$ (with orientation $D$ ) into the rational numbers. $(D, \varphi)$ is a nowhere-zero $\frac{q}{p}+1$-flow on $G=(V, E)$ if $1 \leq \varphi(e) \leq \frac{q}{p}$ for all $e \in E$ and equation (1) is satisfied for all $v \in V$. The circular flow number $F_{c}(G)$ of $G$ is the minimum number $r$ such that $G$ has a nowhere-zero $r$-flow.

Seymour [6] proved that every bridgeless graph has a nowhere-zero 6-flow. Some methods of this paper can be extended to the study of nowhere-zero $r$-flows on graphs. For instance, it can be proved that $F_{c}(G)<6$ for all bridgeless cubic graphs $G$ with $m_{G}^{*} \geq \frac{3}{2} \omega(G)+1$.

\section{REFERENCES}

1. J.A. Bondy, Balanced Colourings and Graph Orientation, Congressus Numerantium XIV (1975) 109-114 
2. F. Jaeger, Balanced valuations and flows in multigraphs, Proc. Amer. Math. Soc. 55 (1975) 237-242

3. F. JAEger, Nowhere-zero flow problems, in: L. W. Beineke, R. J. Wilson eds., Topics in Graph Theory 3, Academic Press, London (1988) 70-95

4. M. KocHOL, Reduction of the 5-flow conjecture to cyclically 6-edge-connected snarks, J. Comb. Theory, Ser. B 90 (2004) 139-145

5. M. Kochol, Restrictions on smallest counterexamples to the 5-flow conjecture, Combinatorica 26 (2006) 83-89

6. P. D. Seymour, Nowhere-zero 6-flows, J. Comb. Theory, Ser. B 30 (1981) 130-135

7. E. Steffen, Circular flow numbers of regular multigraphs, J. Graph Theory, 36 (2001) 124-134

8. W. T. Tutte, On the embedding of linear graphs in surfaces, Proc. London Math. Soc. Ser. 251 (1949) 474-489

9. W. T. TutTe, A contribution to the theory of chromatic polynomials, Canadian J. Math. 6 (1954) 80-91 